\title{
RANDOM FIXED POINT THEOREMS FOR MEASURABLE MULTIFUNCTIONS IN BANACH SPACES
}

\author{
NIKOLAOS S. PAPAGEORGIOU ${ }^{1}$
}

\begin{abstract}
In this paper we prove several random fixed point theorems for measurable closed and nonclosed valued multifunctions satisfying general continuity conditions. Our work extends and sharpens earlier results by Engl, Itoh and Reich.
\end{abstract}

1. Preliminaries and definitions. The study of random fixed points was initiated by the Prague school of probabilists in the fifties. Recently the interest on this subject was revived especially after the survey article of Bharucha-Reid [3]. The theory of random fixed points has found important applications in random operator equations [2], random differential equations in Banach spaces [15] and differential inclusions [4].

Throughout this paper let $(\Omega, \Sigma, \mu)$ be a complete $\sigma$-finite measure space and $X$ a separable Banach space. Additional assumptions will be introduced as needed. By $X^{*}$ we will denote the topological dual of $X$. We will use the following notation: $P_{f(c)}(X)=\{A \subseteq X$ : nonempty, closed, (convex) $\}$ and $P_{(w) k(c)}(X)=$ $\{A \subseteq X$ : nonempty, (weakly)-compact, (convex)\}. If $A \subseteq X$ nonempty, we set $|A|=\sup _{x \in A}\|x\|$. Also by $\sigma_{A}(\cdot)$ we denote the support function of $A$, i.e. $\sigma_{A}\left(x^{*}\right)=$ $\sup _{x \in A}\left(x^{*}, x\right)$ for all $x^{*} \in X^{*}$ and by $d_{A}(\cdot)$ the distance function from $A$, i.e. $d_{A}(x)=\inf _{z \in A}\|x-z\|$ for all $x \in X$. A multifunction $F: \Omega \rightarrow P_{f}(X)$ is said to be measurable if it satisfies any of the following equivalent conditions:

(i) $\operatorname{Gr} F=\{(\omega, x) \in \Omega \times X: x \in F(\omega)\} \in \Sigma \times B(X)$ where $B(X)$ is the Borel $\sigma$-field of $X$,

(ii) $\omega \rightarrow d_{F(\omega)}(x)$ is measurable for all $x \in X$, and

(iii) there exists a sequence $\left\{f_{n}(\cdot)\right\}_{n \geq 1}$ of measurable selectors of $F(\cdot)$ s.t. $F(\omega)=$ $\operatorname{cl}\left\{f_{n}(\omega)\right\}_{n \geq 1}$ (Castaing's representation).

If $(\Omega, \Sigma)$ is a measurable space, then (ii) $\Leftrightarrow$ (iii) $\Rightarrow$ (i).

For details see $[4,13,22]$. By $S_{F}^{1}$ we denote the set of all $L_{X}^{1}(\Omega)$ selectors of $F(\cdot)$, i.e. $S_{F}^{1}=\left\{f(\cdot) \in L_{X}^{1}(\Omega): f(\omega) \in F(\omega) \mu\right.$-a.e. $\}$. Clearly this is a closed subset $L_{X}^{1}(\Omega)$ (maybe empty) and is nonempty if and only if $\inf _{x \in F(\omega)}\|x\| \in L_{+}^{1}(\Omega)$. Let $G: \Omega \rightarrow$ $P_{f}(X)$. A multifunction $F: \operatorname{Gr} G \rightarrow P_{f}(X)$ is called "measurable multifunction with stochastic domain $G(\cdot)$ " if, for all $x \in X$ and all $U \subseteq X$ open, $\{\omega \in \Omega: x \in G(\omega)$ and $F(\omega, x) \cap U \neq \varnothing\} \in \Sigma$. A measurable function $x: \Omega \rightarrow X$ s.t. for $\mu$-almost all $\omega \in \Omega$, $x(\omega) \in G(\omega)$ and $x(\omega) \in F(\omega, x(\omega))$ is said to be a random fixed point for $F(\cdot, \cdot)$. If $Y$ is a topological space $F: Y \rightarrow P_{f}(X)$ is called upper (lower) semicontinuous

Received by the editors October 18, 1984 and, in revised form, February 19, 1985, July 11, 1985 and July 23, 1985.

1980 Mathematics Subject Classification. Primary 60H25, 47H10.

Key words and phrases. Measurable multifunction, stochastic domain, upper hemicontinuous, measurable selector, Hausdorff metric, lower semicontinuous.

${ }^{1}$ Research supported by N.S.F. Grant DMS-8403135. 
if, for any closed (open) subset $A$ of $X, F^{-}(A)=\{y \in Y: F(y) \cap A \neq \varnothing\}$ is closed (open). $F(\cdot)$ is continuous if it is both upper and lower semicontinuous. The multifunction $F(\cdot)$ is said to be upper hemicontinuous if and only if, for all $x^{*} \in X^{*}, y \rightarrow \sigma_{F(y)}\left(x^{*}\right)$ is u.s.c. From [1, Proposition 1, p. 68] we know that every u.s.c. multifunction is upper hemicontinuous. Let $2^{X}=$ nonempty subsets of $X$ \}. Finally one of our basic analytical tools will be Aumann's selection theorem, which for the convenience of the reader we state here in its most general form due to Saint-Beuve [23, Proposition 3, p. 117]. Recall that a $\sigma$-field $\Sigma$ is complete if $\Sigma=\hat{\Sigma}=$ the universal $\sigma$-field, and a Souslin space is a Hausdorff topological space $X$ such that there exists a Polish space (i.e. a complete, separable, metric space) $P$ and a continuous surjection $p: P \rightarrow X$. (For details see [23].)

AUMANN'S THEOREM [23]. Let $(\Omega, \Sigma)$ be a complete measurable space, $X$ a Souslin space with its Borel $\sigma$-field $B(X)$ and $F: \Omega \rightarrow 2^{X}$ a multifunction s.t. Gr $F \in \Sigma \times B(X)$. Then $F(\cdot)$ admits a measurable selector.

REMARK. If $(\Omega, \Sigma, \mu)$ is a complete $\sigma$-finite measure space, then $\Sigma=\hat{\Sigma}$. Also a separable Banach space with its weak topology is a Souslin space. Hence Souslin spaces need not be metrizable, but they are always separable.

2. Main results. We start with a random fixed point theorem that generalizes significantly Theorem 3.1 of Itoh [15] which was stated for compact, convex valued continuous multifunctions.

So assume that $X^{*}$ is separable too and that $K \in P_{k c}(X)$.

THEOREM 1. If $F: \Omega \times K \rightarrow P_{f c}(X)$ is a multifunction with bounded values s.t.,

(1) $(\omega, x) \rightarrow F(\omega, x)$ is measurable,

(2) for all $\omega \in \Omega, F(\omega, \cdot)$ is upper hemicontinuous, and

(3) for each $(\omega, x) \in \Omega \times K, F(\omega, x) \cap K \neq \varnothing$, then $F(\cdot, \cdot)$ has a random fixed point.

PROOF. Consider the multifunction $R: \Omega \rightarrow 2^{K}$ defined by $R(\omega)=\{x \in K: x \in$ $F(\omega, x)\}$. From Theorem 1.16 of Lassonde [19], we have that $R(\omega) \neq \varnothing$ for all $\omega \in \Omega$. Since $F(\cdot, \cdot)$ is closed, convex-valued we can write that

$$
R(\omega)=\bigcap_{x^{*} \in X^{*}}\left\{x \in K:\left(x^{*}, x\right) \leq \sigma_{F(\omega, x)}\left(x^{*}\right)\right\} .
$$

Because of the boundedness of the values of $F(\cdot, \cdot)$ we have that $\sigma_{F(\omega, x)}(\cdot)$ is norm continuous for all $\omega \in \Omega$ and all $x \in X$. Thus if $\left\{x_{n}^{*}\right\}_{n \geq 1}$ is a countable dense subset of $X^{*}$, we can write that

$$
R(\omega)=\bigcap_{n \geq 1}\left\{x \in K:\left(x_{n}^{*}, x\right) \leq \sigma_{F(\omega, x)}\left(x_{n}^{*}\right)\right\}=\bigcap_{n \geq 1} L_{n}(\omega) .
$$

Applying the Castaing representation result on $(\omega, x) \rightarrow F(\omega, x)$ we can see that, for all $n \geq 1,(\omega, x) \rightarrow \sigma_{F(\omega, x)}\left(x_{n}^{*}\right)$ is measurable. Hence $\operatorname{Gr} R=\bigcap_{n \geq 1} \operatorname{Gr} L_{n} \in$ $\Sigma \times B(K)$. Apply Aumann's selection theorem to get $x: \Omega \rightarrow K$ measurable s.t. for all $\omega \in \Omega, x(\omega) \in R(\omega)$. Then clearly $x(\omega) \in F(\omega, x(\omega))$ for all $\omega \in \Omega$, i.e. $x(\cdot)$ is a random fixed point for $F(\cdot, \cdot)$. Q.E.D. 
We have an analogous result for lower semicontinuous multifunctions. Now assume that $K \in P_{w k c}(X)$. Recall that $f: \Omega \times X \rightarrow X$ is a Carathéodory function if it is measurable with respect to $\omega$, and continuous with respect to $x$.

THEOREM 2. If $F: \Omega \times K \rightarrow P_{f c}(K)$ is a mulifunction s.t.,

(1) $F(\cdot, \cdot)$ is $\Sigma \times B(X)$-measurable,

(2) for all $\omega \in \Omega, F(\omega, \cdot)$ is l.s.c. from $X_{w}$ into $X$, then $F(\cdot, \cdot)$ has a random fixed point.

ProOF. Recall that since $X$ is separable, $K$ with the weak topology is a compact metrizable space (see Dunford and Schwartz [9, Theorem 3, p. 434]). Also since $\Sigma$ is $\mu$-complete, assumption $A$ of Fryszkowski [11] is satisfied (Von Neumann's projection theorem, see Saint-Beuve [23, Theorem 4, p. 121]). These facts together with hypothesis (1) and the lower semicontinuity of $F(\omega, \cdot)$, for all $\omega \in \Omega$, allow us to apply Theorem 1 of $[\mathbf{1 1}]$ and find a Carathéodory function $f: \Omega \times K_{w} \rightarrow K$ s.t., for all $(\omega, x) \in \Omega \times K$, we have $f(\omega, x) \in F(\omega, x)$. So we can apply Theorem 2.1 of Itoh [15] and conclude that $f(\cdot, \cdot)$ has a random fixed point $x: \Omega \rightarrow X$. Then $x(\omega)=f(\omega, x(\omega)) \in F(\omega, x(\omega))$ for all $\omega \in \Omega$. Q.E.D.

Now we pass to another important class of multifunctions, namely the concave ones. Recall that $F: X \rightarrow 2^{X}$ is said to be concave if for all $x_{1}, x_{2} \in X$ and all $\lambda \in[0,1] \lambda F\left(x_{1}\right)+(1-\lambda) F\left(x_{2}\right) \subseteq F\left(\lambda x_{1}+(1-\lambda) x_{2}\right)$. Multifunctions of this kind appear often in mathematical economics.

Assume that $(\Omega, \Sigma, \mu)$ is a complete finite measure space and $X$ a separable Banach space.

THEOREM 3. If $F: \Omega \times X \rightarrow P_{w k c}(X)$ is a multifunction s.t.

(1) $(\omega, x) \rightarrow F(\omega, x)$ is measruable and $F(\omega, x) \subseteq G(\omega) \mu$-a.e. where $G: \Omega \rightarrow$ $P_{w k c}(X)$ is integrably bounded,

(2) for all $\omega \in \Omega, F(\omega, \cdot)$ is upper hemicontinuous and concave, then $F(\cdot, \cdot)$ admits a random fixed point.

Proof. Since $G(\cdot)$ is integrably bounded and $P_{w k c}(X)$-valued from Theorem 4.2 of [24] we know that $S_{G}^{1}$ is a $w$-compact subset of $L_{X}^{1}(\Omega)$. Consider the setvalued operator $T: S_{G}^{1} \rightarrow P_{w k c}\left(S_{G}^{1}\right)$ defined by $T(x)=S_{F(\cdot, x(\cdot))}^{1}$. We claim that it is $w$-u.s.c. For all $p(\cdot) \in\left[L_{X}^{1}(\Omega)\right]^{*}=L_{X_{w^{*}}^{*}}^{\infty}(\Omega)$ (see $[26$, p. 95]) we have

$$
\begin{aligned}
\sigma_{T(x)}(p) & =\sigma_{S_{F(\cdot, x(\cdot))}^{1}}(p)=\sup _{f \in S_{F(\cdot, x(\cdot))}^{1}}\langle p, f\rangle \\
& =\sup _{f \in S_{F(\cdot, x(\cdot))}^{1}} \int_{\Omega}(p(\omega), f(\omega)) d \mu(\omega) .
\end{aligned}
$$

Using Theorem 2.2 of Hiai and Umegaki $[\mathbf{1 2}]$ we have that

$$
\begin{aligned}
\sup _{f \in S_{F(\cdot, x(\cdot))}^{1}} \int_{\Omega}(p(\omega), f(\omega)) d \mu(\omega) & =\int_{\Omega} \sup _{z \in F(\omega, x(\omega))}(p(\omega), z) d \mu(\omega) \\
& \Rightarrow \sigma_{T(x)}(p)=\int_{\Omega} \sigma_{F(\omega, x(\omega))}(p(\omega)) d \mu(\omega) .
\end{aligned}
$$

Because of the concavity of $F(\omega, \cdot)$ it is easy to see that $\sigma_{F(\omega, \cdot)}(p(\omega))$ is concave. So $x(\cdot) \rightarrow \sigma_{S_{F(\cdot, x(\cdot))}^{1}}(p)$ is concave. We will show that it is also $w$-u.s.c. Because of 
concavity it suffices to show that it is u.s.c. So we need to show that $U_{\lambda}=\{x(\cdot) \in$ $\left.L_{X}^{1}(\Omega): \sigma_{S_{F(\cdot, x(\cdot))}^{1}}(p) \geq \lambda\right\}$ is closed. So let $\left\{x_{n}(\cdot)\right\}_{n \geq 1} \subseteq U_{\lambda}$ and $x_{n}(\cdot) \stackrel{L_{X}^{1}}{\rightarrow} x(\cdot)$. By passing to a subsequence if necessary we may assume that $x_{n}(\omega) \stackrel{s}{\rightarrow} x(\omega) \mu$-a.e. So we have

$$
\begin{aligned}
\varlimsup_{n \rightarrow \infty} \sigma_{S_{F\left(\cdot, x_{n}(\cdot)\right)}^{1}}(p) & \leq \int_{\Omega} \varlimsup \sigma_{F\left(\omega, x_{n}(\omega)\right)}(p(\omega)) d \mu(\omega) \\
& \leq \int_{\Omega} \sigma_{F(\omega, x(\omega))}(p(\omega)) d \mu(\omega) .
\end{aligned}
$$

The last inequality follows from the fact that, for all $\omega \in \Omega, F(\omega, \cdot)$ is by hypothesis upper hemicontinuous. Hence

$$
\lambda \leq \int_{\Omega} \sigma_{F(\omega, x(\omega))}(p(\omega)) d \mu(\omega)=\sigma_{S_{F(\cdot, x(\cdot))}^{1}}(p) \Rightarrow x(\cdot) \in U_{\lambda} .
$$

Thus $\sigma_{T(\cdot)}(p)$ is $w$-u.s.c. and so Theorem II-20 of [4] tells us that $T(\cdot)$ is $w$-u.s.c. Applying Theorem 2 of Himmelberg [14] we deduce that $T(\cdot)$ admits a fixed point $\hat{x}(\cdot) \in S_{G}^{1}$. Then

$$
\hat{x} \in T(\hat{x}) \Rightarrow \hat{x}(\cdot) \in S_{F(\cdot, \hat{x}(\cdot))}^{1} \Rightarrow \hat{x}(\omega) \in F(\omega, \hat{x}(\omega)) \quad \mu \text {-a.e. Q.E.D. }
$$

In [18] Ky Fan proved an important result that combined fixed point theory with the study of proximity maps. This result was extended further by Reich [21] and found several important applications. Here we present a more general stochastic version of it. Let $X$ be a separable reflexive Banach space. Recall that if $A$ is a nonempty subset of $X$, the metric projection map $P_{A}(\cdot): X \rightarrow 2^{A} \cup\{\varnothing\}$ is defined by $P_{A}(x)=\left\{z \in A: d_{A}(x)=\|x-z\|\right\}$. Also by $s$ we will denote the strong topology on $X$.

THEOREM 4. If $F: \Omega \rightarrow P_{w k c}(X)$ is a measurable multifunction s.t., for all $\omega \in \Omega, s$-int $F(\omega) \neq \varnothing$ and if $f: \mathrm{Gr} F \rightarrow X$ is a continuous random operator with stochastic domain $F(\cdot)$, then there exists $x(\cdot)=$ measurable selector of $F(\cdot)$ s.t., for all $\omega \in \Omega$,

$$
\|x(\omega)-f(\omega, x(\omega))\|=d_{F(\omega)}(f(\omega, x(\omega))) .
$$

PROOF. Consider the metric projection multifunction $p_{F(\cdot)}(\cdot)$. From [21, Proposition 2.1] we know that, for all $\omega \in \Omega, x \rightarrow P_{F(\omega)}(x)$ is u.s.c. By definition, $P_{F(\omega)}(x)=\left\{z \in X: d_{F(\omega)}(x)=\|z-x\|\right\} \cap F(\omega)$. Since $F(\cdot)$ is $P_{w k c}(X)$-valued, $P_{F(\omega)}(x) \neq \varnothing$ for all $(\omega, x) \in \Omega \times X$. Also note that for all $x \in X,(\omega, z) \rightarrow$ $d_{F(\omega)}(x)-\|z-x\|$ is a Carathéodory function and so it is jointly measurable (see [4, Lemma III-14, p. 70]). Hence for all $x \in X, \operatorname{Gr} P_{F(\cdot)}(x)=\{(\omega, z) \in$ $\left.\Omega \times X: d_{F(\omega)}(x)-\|z-x\|=0\right\} \cap \operatorname{Gr} F \in \Sigma \times B(X)$ and since $P_{F(\cdot)}(x)$ is closed valued, we deduce that, for all $x \in X, \omega \rightarrow P_{F(\omega)}(x)$ is measurable.

Now consider the composite multifunction

$$
R(\omega, x)=\left(P_{F} \circ f\right)(\omega, x)=P_{F(\omega)}(f(\omega, x)) .
$$

So $R(\cdot, \cdot)$ is a multifunction with stochastic domain $F(\cdot)$. We will show that it is measurable. Let $K \subseteq X$ be nonempty, closed. Then $\{\omega \in \Omega: x \in F(\omega), R(\omega, x) \cap$ $\left.K=P_{F(\omega)}(f(\omega, x)) \cap K \neq \varnothing\right\}=\left\{\omega \in \Omega: x \in F(\omega), f(\omega, x) \in P_{F(\omega)}^{-}(K)\right\}$. Since for all $\omega \in \Omega, P_{F(\omega)}(\cdot)$ is u.s.c. and we know that $P_{F(\omega)}^{-}(K)$ is closed. Hence 
$\left\{\omega \in \Omega: x \in F(\omega), f(\omega, x) \in P_{F(\omega)}^{-}(K)\right\} \in \Sigma$, which means that $R(\cdot, \cdot)$ is indeed measurable. Furthermore, for fixed $\omega \in \Omega, R(\omega, \cdot)$ being the composition of two u.s.c. multifunctions is itself u.s.c. Finally, $\bigcup_{x \in F(\omega)} R(\omega, x) \subseteq F(\omega)$. So we can apply Theorem 7 of Engl [10] and get that there exists $x(\cdot)$ measurable selector of $F(\cdot)$ s.t. $x(\omega) \in R(\omega, x(\omega))$ for all $\omega \in \Omega$. Hence $x(\omega) \in P_{F(\omega)}(f(\omega, x(\omega))) \Rightarrow$ $\|x(\omega)-f(\omega, x(\omega))\|=d_{F(\omega)}(f(\omega, x(\omega)))$ for all $\omega \in \Omega$. Q.E.D.

REMARK. In his assumptions in Theorem 7, Engl [10] includes the existence of a measurable selector $x(\cdot)$ for int $F(\cdot)$. This is redundant, thanks to Lemma 4 of Cornwall [5] and the Aumann selection theorem.

Next we will prove a random fixed point theorem for nonclosed-valued multifunctions. We believe that this theorem will be useful to mathematical economists who deal with preference multifunctions that describe the "better than" set for each individual agent. By its nature this multifunction cannot be closed valued. Also the theorem can be useful in solving differential inclusions. We will need the following auxiliary Lemma. Assume that $X, Y$ are locally compact, metric spaces and $f: \Omega \times X \rightarrow Y$. By $C(X, Y)$ we denote the space of continuous functions from $X$ into $Y$ endowed with the compact-open topology. If $X=Y$ we simply write $C(X)$.

LEMMA. $f(\cdot, \cdot)$ is a Carathéodory function if and only if $\omega \rightarrow r(\omega)(\cdot)=f(\omega, \cdot)$ is a measurable function from $\Omega$ into $C(X, Y)$.

Proof. First assume that $f(\cdot, \cdot)$ is Carathéodory. Let $B$ be a basis element for $C(X, Y)$ with compact-open topology. Then $B=\{g(\cdot) \in C(X, Y): g(K) \subseteq V\}$ where $K \subseteq X$ is compact, $V \subseteq Y$ is open. We need to show that $r^{-1}(B) \in \Sigma$. Let $\left\{x_{n}\right\}_{n \geq 1}$ be dense in $K$. Then we have

$$
\begin{aligned}
r^{-1}(B) & =\{\omega \in \Omega: r(\omega)(\cdot) \in B\}=\{\omega \in \Omega: r(\omega)(K) \subset V\} \\
& =\{\omega \in \Omega: f(\omega, K) \subseteq V\}=\bigcap_{n \geq 1}\left\{\omega \in \Omega: f\left(\omega, x_{n}\right) \in V\right\} \in \Sigma
\end{aligned}
$$

since by hypothesis $f(\cdot, \cdot)$ is Carathéodory.

Now assume that $\omega \rightarrow r(\omega)(\cdot)=f(\omega, \cdot)$ is measurable from $\Omega$ into $C(X, Y)$.

Let $(r$, Id $): \Omega \times X \rightarrow C(X, Y) \times X$ be defined by $(r$, Id $)(\omega, x)=(r(\omega)(\cdot), x)$. Clearly this is measurable. Let $e$ be the evaluation map on $C(X, Y) \times X$. From Dugundji $[8$, Theorem 2.4(b), p. 260] we know that $e(\cdot, \cdot)$ is continuous. Consider the map $u: \Omega \times X \rightarrow Y$. defined by $u=e \circ[(r, \mathrm{Id})]$. Then $u(\omega, x)=e[(r(\omega)(\cdot), x)]=$ $r(\omega)(x)=f(\omega, x) \Rightarrow u(\cdot, \cdot)=f(\cdot, \cdot)$. But $u(\cdot, \cdot)$ is a Carathéodory function. Hence so is $f(\cdot, \cdot)$. Q.E.D.

Now we are ready for the random fixed point theorem for nonclosed valued multifunctions. Assume that $X$ is a finite-dimensional Banach space. Such a space is automatically separable and locally compact, (see [16]).

THEOREM 5. If $F: \Omega \times X \rightarrow 2^{X}$ is a multifunction with bounded convex values with nonempty interior s.t.

(1) for all $x \in X, \operatorname{Gr} F(\cdot, x) \in \Sigma \times B(X)$,

(2) for all $\omega \in \Omega, F(\omega, \cdot)$ is continuous for the Hausdorff pseudometric and $F(\omega, X)$ is bounded, then $F(\cdot, \cdot)$ has a random fixed point. 
PROOF. Using the properties of the Hausdorff metric it is easy to see that int $F(\omega, \cdot)$ is continuous for the Hausdorff pseudometric and so, in particular, it is l.s.c. So if we consider the multifunction $R(\cdot)$ from $\Omega$ into the subsets of $C(X)$ defined by $R(\omega)=\{f(\cdot) \in C(X): f(x) \in \operatorname{int} F(\omega, x)$ for all $x \in X\}$, Thenrem $3.1^{\prime \prime \prime}(\mathrm{c})$ of Michael [20] tells us that, for all $\omega \in \Omega, R(\omega) \neq \varnothing$. If by $\partial F(\omega, x)$ we denote the boundary of the set $F(\omega, x)$, then we can rewrite $R(\cdot)$ as follows: $R(\omega)=\left\{f(\cdot) \in C(X): d_{\partial F(\omega, x)}(f(x))>0\right.$ for all $\left.x \in X\right\} \cap \operatorname{cs}(F(\omega, \cdot))$. From hypothesis (2) we have that $\overline{F(\omega, \cdot)}$ is Hausdorff continuous. Hence Proposition 2.1 of [6] tells us that $\partial \overline{F(\omega, \cdot)}$ is Hausdorff continuous. But $\partial \overline{F(\omega, \cdot)}=\partial F(\omega, \cdot)$. Thus, $\partial F(\omega, \cdot)$ is Hausdorff continuous. Now given $\varepsilon>0$ let $\delta>0$ be s.t. if $x^{\prime} \in B_{\delta}(x)$, $h\left(\partial F(\omega, x), \partial F\left(\omega, x^{\prime}\right)\right)<\varepsilon / 2$ and $\left\|f(x)-f\left(x^{\prime}\right)\right\|<\varepsilon / 2$. Then

$$
\begin{aligned}
& \left|d_{\partial F(\omega, x)}(f(x))-d_{\partial F\left(\omega, x^{\prime}\right)}\left(f\left(x^{\prime}\right)\right)\right| \\
& \quad \leq\left|d_{\partial F(\omega, x)}(f(x))-d_{\partial F\left(\omega, x^{\prime}\right)}(f(x))\right|+\left|d_{\partial F\left(\omega, x^{\prime}\right)}(f(x))-d_{\partial F\left(\omega, x^{\prime}\right)}\left(f\left(x^{\prime}\right)\right)\right| \\
& \quad \leq h\left(\partial F(\omega, x), \partial F\left(\omega, x^{\prime}\right)\right)+\left\|f(x)-f\left(x^{\prime}\right)\right\|<\varepsilon / 2+\varepsilon / 2=\varepsilon .
\end{aligned}
$$

So $x \rightarrow d_{\partial F(\omega, x)}(f(x))$ is continuous. Also Theorem 4.6(iv) of [13] tells us that $\omega \rightarrow \partial F(\omega, x)$ is measurable, hence $\omega \rightarrow d_{\partial F(\omega, x)}(f(x))$ is measurable. Therefore, $(\omega, x) \rightarrow d_{\partial F(\omega, x)}(f(x))$ is a Carathéodory function. So the lemma tells us that $\omega \rightarrow d_{\partial F(\omega, \cdot)}(g(\cdot))$ is measurable from $\Omega$ into $C(X, \mathbf{R})$. Now consider the map $L: \Omega \times C(X) \rightarrow C(X, \mathbf{R})$ defined by $L(\omega, g(\cdot))=d_{\partial F(\omega, \cdot)}(g(\cdot))$. We just saw that $\omega \rightarrow L(\omega, g(\cdot))$ is measurable. Also since the distance function is Lipschitz and in our case the compact open topology and the topology of uniform convergence on compact sets coincide, we deduce that $g(\cdot) \rightarrow L(\omega, g(\cdot))$ is continuous. So $L(\cdot, \cdot)$ is a Carathéodory function. Since $C(X)$ is separable, metrizable (see $[\mathbf{1 7}$, Theorems 1 and 3, pp. 93-94]) Lemma III-14, p. 70 of $[4]$ tells us that $L(\cdot, \cdot)$ is $\Sigma \times B(C(X))$ measurable. Then $\operatorname{Gr} R=\{(\omega, g(\cdot)) \in \Sigma \times C(X): L(\omega, g(\cdot)) \in$ int $\left.C_{+}(X, \mathbf{R})\right\} \cap \operatorname{Gr}(\operatorname{cs}(F(\omega, \cdot))) \in \Sigma \times B(C(X))$. Applying Aumann's theorem we can find $r: \Omega \rightarrow C(X)$ measurable s.t. $r(\omega) \in R(\omega)$ for all $\omega \in \Omega$. Set $r(\omega)(\cdot)=$ $f(\omega, \cdot)$. The lemma tells us that $f(\cdot, \cdot)$ is a Carathéodory selector of $F(\cdot, \cdot)$. Since $f(\omega, X) \subseteq F(\omega, X)=$ bounded we have that, for all $\omega \in \Omega, f(\omega, \cdot)$ has a relatively compact range. So we can apply Corollary 2.2 of $[15]$ and conclude that $f(\cdot, \cdot)$ has a random fixed point $x(\cdot)$. Clearly this is a random fixed point for $F(\cdot, \cdot)$ too. Q.E.D.

REMARK. In the core of the above proof lies what we believe to be an interesting Carathéodory selection theorem for nonclosed valued multifunctions, which partially generalizes Theorem 1 of Fryszkowski [11].

We will close this note by proving a random fixed point theorem important class of multifunction, namely contraction multifunctions with stochastic domain. Our result generalizes Corollary 15 of Engl [10]. So $X$ is a separable Banach space and $h(\cdot, \cdot)$ denotes the Hausdorff metric.

THEOREM 6. If $G: \Omega \rightarrow P_{f}(x)$ is integrably bounded and $F: \operatorname{Gr} F \rightarrow P_{f}(x)$ is a measurable multifunction with stochastic domain s.t. for all $\omega \in \Omega$ and all $x, y \in G(\omega), h(F(\omega, x), F(\omega, y)) \leq k(\omega)\|x-y\|$ where $\|k\|_{\infty}<1$, then $F(\cdot, \cdot)$ has a random fixed point. 
PROOF. Consider the set-valued operator $T: S_{G}^{1} \rightarrow P_{f}\left(L_{X}^{1}(\Omega)\right)$ defined by $x(\cdot) \rightarrow$ $S_{F(\cdot, x(\cdot))}^{1}$. For $x_{1}(\cdot), x_{2}(\cdot) \in S_{G}^{1}$ we have

$$
\begin{aligned}
h\left(T x_{1}, T x_{2}\right) & =\sup _{\|g\|_{\infty} \leq 1}\left|\sigma_{S_{F(\cdot, x(\cdot))}^{1}}(g)-\sigma_{S_{F\left(\cdot, x_{2}(\cdot)\right)}^{1}}(g)\right| \\
& =\sup _{\|g\|_{\infty}}\left|\int_{\Omega}\left(\sigma_{F\left(\omega, x_{2}(\omega)\right)}(g(\omega))-\sigma_{F\left(\omega, x_{2}(\omega)\right)}(g(\omega))\right) d \mu(\omega)\right| \\
& \leq \sup _{\|g\|_{\infty} \leq 1} \int_{\Omega}\left|\sigma_{F\left(\omega, x_{1}(\omega)\right)}(g(\omega))-\sigma_{F\left(\omega, x_{2}(\omega)\right)}(g(\omega))\right| d \mu(\omega) \\
& =\int_{\Omega\left\|x^{*}\right\| \leq 1} \sup _{F\left(\omega, x_{1}(\omega)\right)}\left(x^{*}\right)-\sigma_{F\left(\omega, x_{2}(\omega)\right)}\left(x^{*}\right) \mid d \mu(\omega) \\
& =\int_{\Omega} h\left(F\left(\omega, x_{1}(\omega)\right), F\left(\omega, x_{2}(\omega)\right) d \mu(\omega) \leq\|k\|_{\infty}\left\|x_{1}-x_{2}\right\|_{1} .\right.
\end{aligned}
$$

So $T(\cdot)$ is a contraction multifunction. Nadler's theorem [25] tells us that $T(\cdot)$ admits a fixed point. So there exists $x(\cdot) \in S_{G}^{1}$ s.t. $x \in T x \Rightarrow x(\omega) \in F(\omega, x(\omega))$ $\mu$-a.e. and $x(\omega) \in G(\omega) \mu$-a.e., i.e. $x(\cdot)$ is a random fixed point for $F(\cdot, \cdot)$. Q.E.D.

ACKNOWLEDGMENT. The author is very grateful to the referee for his invaluable comments and suggestions that improved this work significantly.

\section{REFERENCES}

1. J. P. Aubin, Mathematical methods in game and economic theory, North-Holland, Amsterdam, 1979.

2. A. T. Bharucha-Reid, Random integral equations, Academic Press, New York, 1972.

3. __ Fixed point theorems in probabilistic analysis, Bull. Amer. Math. Soc. 82 (1976), 641-645.

4. C. Castaing and M. Valadier, Convex analysis and measurable multifunctions, Lecture Notes in Math., Vol. 580, Springer-Verlag, Berlin, 1977.

5. R. Cornwall, Conditions for the graph and the integral of a correspondence to be open, J. Math. Anal. Appl. 39 (1972), 771--792.

6. F. DeBlasi and G. Pianigiani, Remarks on Hausdorff continuous multifunctions and selections, Comm. Math. Univ. Carolin. 24 (1983), 553-561.

7. J. Diestel and J. Uhl, Vector measures, Math. Surveys, Vol. 15, Amer. Math. Soc., Providence, R.I., 1977.

8. J. Dugundji, Topology, Allyn and Bacon, Boston, Mass., 1966.

9. N. Dunford and J. T. Schwartz, Linear operators. I, Wiley Interscience, New York, 1958.

10. H. Engl, Random fixed point theorems for multivalued mappings, Pacific J. Math. 76 (1976), 351-360.

11. A. Fryszkowski, Caratheodory type selectors of set valued maps of two variables, Bull. Acad. Polon. Sci. Sér. Sci. Math. 25 (1977), 41-46.

12. F. Hiai and H. Umegaki, Integrals, conditional expectations and martingales of multivalued functions, J. Multivariate Anal. 7 (1977), 149-182.

13. C. Himmelberg, Measurable relations, Fund. Math. 87 (1975), 53-72.

14. , Fixed points of compact multifunctions, J. Math. Anal. Appl. 38 (1972), 205-207.

15. S. Itoh, Random fixed point theorems with an application to random differential equations in Banach spaces, J. Math. Anal. Appl. 67 (1979), 261-273.

16. K. Kuratowski, Topology. I, Academic Press, New York, 1966.

17. , Topology. II, Academic Press, New York, 1966.

18. Ky Fan, Extensions of two fixed point theorems of F. Browder, Math. Z. 112 (1969), 234-240. 
19. M. Lassonde, On the use of K.K.M. multifunctions in fixed point theory and related topics, J. Math. Anal. Appl. 97 (1983), 151-201.

20. E. Michael, Continuous selections. I, Ann. of Math. 63 (1965), 361-382.

21. S. Reich, Approximate selections, best approximations, fixed points and invariant sets, J. Math. Anal. Appl. 62 (1978), 104-112.

22. R. T. Rockafellar, Measurable dependence of convex sets and functions on parameters, J. Math. Anal. Appl. 28 (1969), 4-25.

23. M. Saint-Beuve, On the extension of Von Neumann-Aumann's theorem, J. Funct. Anal. 17 (1974), 112-129.

24. N. S. Papageorgiou, Representation of set valued operators, Trans. Amer. Math. Soc. 292 (1985), 557-572.

25. S. B. Nalder, Multivalued contraction mappings, Pacific J. Math. 30 (1969), 476-488.

26. A. Ionescu-Tulcea and C. Ionescu-Tulcea, Topics in the theory of lifting, Springer-Verlag, Berlin, 1969.

Department of MAThematics, University of Illinois, 1409 W. Green Street, URBANA, ILLINOIS 61801

Current address: University of Thessaloniki, School of Technology, Mathematics Division, Thessaloniki 54006, Greece 